\title{
Rudolf Staehelin † 28. August 1875-26. März 1943
}

Prof. Dr. Rudolf Staehelin ist uns am 26. März 1943 plötzlieh ent-rissen worden. Ein Herzleiden, das ihn vor einem Jahr auf das Kranken-lager geworfen, von dem er sich aber auffallend gut erholt hatte, hat ihn dahingerafft, schmerzlos und mitten aus der Arbeit - ein herber Verlust für uns, die ihn als Kliniker und als Arzt verehrten und als Freund lieb hatten; denn Männern wie Rudolf Staehelin begegnet man nicht oft. , „Höchstes Glück der Erdenkinder sei nur die Persönlichkeit”, und dies war ihm gegeben. Aufgewachsen als Sohn eines bedeutenden Vaters, des hervorragenden Theologen, und einer feinsinnigen Mutter, genoß er im Gymnasium ausgezeichneten humanistischen Unterrícht

und wurde dann auf der Basler Iloehschule durch die besten Lehrer in die Medizin eingeführt. So konnte sich der Jüngling, dem ein gütiges Geschick die schönsten Geistesgaben in die Wiege gelegt hatte -, Intelligenz, Verstand, Beobaehtung und Arbeitseifer - zu einem cha- raktervollen und gebildeten Menschen entwickeln. Staehelin hatte sich schon früh eine philosophisehgesehichtliehe Basis angeeignet, die ihm im späteren Leben ermöglichte, medizinische Probleme mit weitem Buck zu fassen. Selbst anscheinend nebensächliches entging ihm nicht, doch blieb er nie dabei stehen, sondern er strebte, den Allgemeinsinn der biologischen Erscheinungen induktiv zu begreifen. Analyse und Syn-these waren bei ihm selbstverständlich und eng verbunden. Unter dem Anatomen Kollmann hat er früh gelernt, die Körperformen plastisch zu sehen, und beim Pathologen Roth hat er solide Kenntnisse in der Mor- phologie der Krankheiten gesammelt. Ausgezeichnet vorgebildet erlebte er dann Persönlichkeiten wie Friedrich Müller und hernach Wilhelm His. Unter diesen beiden führenden Geistern ist er gereift und zum Kliniker geworden. Eine an tiefen Eindrücken reiche Jugend! Seine ersten Arbeiten sind den Stoffwechselproblemen gewidmet. Zuerst der Gaswechsel in der Höhe (angeleitet von A. Jaquet), dann Stoff-und Kraftwechsel unter dem Einfluß verschiedener Ernährung beim normalen und beim pankreaslosen Hund (mit Falta und Grote), wo-bei als Ursache der auffallenden Gewichtsabnahme diabetischer Hunde die Steigerung nicht nur des Eiweißzerfalls und des Gesamtumsatzes im Sinne Rubners, sondern auch der Fettverbrennung erkannt wurde. Auch in der Folge ist Staehelin immer wieder Stoff-wechselfragen nachgegangen, hat nachgeí' orscht, wie i,nnere Ursachen (Zwergwuchs, Strumektomie) und äußere (Iníektionskrankheiten, Heil-quellen, vegetarische Nahrung usw.) ihn beeinflussen. Speziell sei die Arbeit über Stoffwechsel im Fieber angeführt, wo er an mit Surratry-panosomen infizierten Hunden eine pathologische Fettemschmelzung feststellen konnte. Aus seiner Klinik stammen die aufschlußreiehen, Arbeiten Loefflers über Grundumsatz bei Morbus Addison, parathy-reopriver Tetanie, Myasthenia gravis pseudo-paralytica, über den Gaswechsel beim Diabetes mellitus nach Zufuhr von reinen Eiweißkörpern und reinen Kohlehydraten, ferner über Desaminierung und Harnstoíf-bildung in der Leber. Ebenso sind die Arbeiten Staubs über Insulin iind Synthalin in Staehelins Klinik durchgeführt worden. Als erfahrener Kliniker hat er später Hochgebirgsphysiologie und klimatisehe Kuren zusammenfassend dargestellt, ferner Stoffwechsel und Sport, Ernährung als Krankheitsursache und Heilfaktor, Diät bei Fettsucht, bei tu-berkulösen Erkrankungen und so manches andere. Genannt seien auch seine Arbeiten über Magenkrankheiten, die seines 
Mitarbeiters Rodella über Darmfäulnis. Seine reichen Erfahrungen in der Ernährungs- und Stoffwechselpathologie hat er nutzbar gemacht bei der Einrichtung einer vorbildlichen Diätküche in seiner Klinik, die sein Schüler Kapp leitet.

Sehr bald hat sich Staehelin auch andern Gebieten der innern Me-dizin zugewandt. Vor allem hat er teilgenommen an der Ausarbeitung und Verfeinerung kliniseher Untersuchungsmethoden, wie der Rönt- genologie und der Elektrocardiographie, die gerade bekannt geworden war, als er naeh Berlin kam. Zur riehtigen Deutung der Röntgen-bilder, namentlich der Thoraxbilder, hat er viel beigetragen. So ist auch aus seiner Schule der ausgezeichnete Basler Röntgenologe Lüdin hervorgegangen. Die Kenntnis der Pathologie des Herzens und des Ge-fäßsystems hat er bereichert durch Untersuchungen über den Blutdruck bei Luftdruckschwankungen (bei Ballonfahrten und in der Unterdruck-kammer), über die Reservekraft des Herzens, über Arythmien. Später sind die hervorragenden Arbeiten von Alois Müller über Hämodynamik an seiner Klinik durchgeführt worden.

Der Bakteriologie wurde an seiner Klinik gründliches Studium ge-widmet; ieh nenne hier die zahlreichen bakteriologischen Untersuchungen (über anaerobe Bakterien, über Influenza, über Sepsis lenta, über Anaphylaxie usw.) seines treuen Mitarbeiters und Freundes Massini. Zu den Lieblingsgebieten gehörten Physiologie und Pathologie der Respirationsorgane. Seine Untersuchungen über Emphysem und 'über die veränderte Atmung bei Asthma, über Lungentumoren, sind grundlegend. Von besonderer Bedeutung sind seine Arbeiten über Tuberkulose. Es kann mit Recht gesagt werden, daß sie mit zum Besten gehören, was über dieses Gebiet geschrieben worden ist. Hier, bei Staehelin, kann sich der Arzt, der Therapeut, den sichersten Rat holen, wie auch der Gutachter, der sich über die heiklen Fragen des Krankheitsbeginnes und der Verantwortung zu äußern hat.

Aber auch auf den Gebieten der Hämatologie und der Neurologic hat Staehelin wertvolle Arbeit geleistet.

Schließlich möchte ich noch die Intoxikationen und gewerblichen Vergiftungen ervvähnen, wie die Massenintoxikationen mit Triortho-kresylphosphat; oder die Aufgaben des Klinikers bei neuen und sel-teneren Vergiftungsbildern (Zangger-Festschrift).

In all diesen vielen Publikationen, die ich nur unvollständig an-führen kann, erkennt man eine leitende Idee, die bei Staehelin schon frühzeitig zur Reife kam: die pathologischen Erscheinungen nicht nur morphologisch, sondern funktionell aufzufassen; in der Klinik nicht nur pathologisch-anatomische Diagnosen zu stellen - obgleich er die pathologische Anatomie beherrschte und niemals vernachlässigte -, sondern das pathologische Geschehen dynamisch zu begreifen. So hat er sehr früh mit Leo Mohr den Plan gefaßt - und auch durchgeführt -, ein Handbuch der innern Medizin herauszugeben, in dem die patho-logisch-physiologische Betrachtungsweise als Grundlage des klinischen Denkens und Handelns zum Ausdruck gebracht wird. Seither hat die „funktionelle Pathologie” viel Erfolg gehabt. Staehelin war einer der ersten, der die Lehren von Cohnheim, von Friedrich Müller und Wil-helm His, seinen Lehrern, wie auch diejenigen Krehls weiter ausge-führt und auf breitester Basis den Ärzten zugänglich gemacht hat. Mit seinen Mitarbeitern hat er den Praktikern eine Nosologie vorgelegt, die von größtem praktischem Nutzen war. So erklärt sich der Erfolg seines mit Mohr und später mit v. Bergmann redigierten Handbuches. Dieser Erfolg ist in hohem Maß den Kapiteln aus seiner Feder zu verdanken, wie seiner meisterhaften Darstellung der Lungenkrankheiten.

Staehelin wurde der Lehrer zahlreicher Ärzte, in gleichem Maße der Studenten, wie der praktischen Ärzte und seiner Kollegen, und so wird er sicherlich auch der Lehrer maneher noch 
kommenden Ge-nerationen bleiben. Er hat eine Schule hinterlassen, die unserm Va-terland Ehre macht. Hingebungsvoll und erfolgreich hat er die Ge-schicke der Schweizerischen Gesellsehaft für innere Medizin und der Schweizerischen Medizin-biologischen Gesellsehaft geleitet.

Es ist nicht möglich, auf beschränktem Raum ein vollständiges Bild dessen zu geben, was alles Staehelin fesselte, ihn anregen konnte und zum Nachdenken brachte. Aber selbst diese kurze Skizze läßt uns staunen wegen der Fülle seiner Ergebnisse, wegen der gründliehen Beobachtung, wegen seines seharfen Verstandes. Wenn sich diese Le-bensarbeit zu einem stolzen Bau gefügt hat, so findet dies seine Be-gründung darin, daß er offenen Sinnes und freudig bereit jeden einzelnen Lebensformen gegenüber stand, daß für ihn Leben Erleben be-deutete. Auf ihn paßt das Wort, daß „Idealismus nur dann groß er-scheint, wenn er über das geredete und geschriebene Wort hinausgeht und durch Selbstaufopferung zur Tat wird”.

So kam Staehelin zu großem Ansehen, in seinem Vaterland und im Ausland, und wir waren froh und stolz zu sehen, wie ihm die Gelehrten, in Frankreich, in Holland, in Schweden, mit hohem Respekt entgegen-kamen. Überall war der bescheidene Mann - bescheiden, weil inner-lich sicher - als einer der besten und klügsten anerkannt.

Auch am Grabe Staehelins können wir aussprechen, was einmal Friedrich Müller von seinem Lehrer Karl Gerhardt gesagt hat: ,,Und wenn wir uns fragten, worin seine Anziehungskraft und Autorität be-gründet waren, so lautet die Antwort: Weil jeder Studierende sofort die innere Wahrhaftigkeit dieses Mannes empfand: hier stand ein Mann, an dem alles echt war, und der nur dasjenige lehrte, woran er selber unbedingt glaubte."

In Wehmut gedenken wir heute des bedeutenden Klinikers, des ausgezeichneten Menschen, des untadeligen Charakters. L. Michaud. 